\title{
Current Understanding of the Progression and Management of Thyroid Associated Orbitopathy: A Systematic Review
}

\author{
Nickisa M. Hodgson · Fatemeh Rajaii (D)
}

Received: September 27, 2019 / Published online: December 10, 2019

(C) The Author(s) 2019, corrected publication 2021

\begin{abstract}
Thyroid associated orbitopathy (TAO) is a common diagnosis encountered by ophthalmologists and oculoplastic surgeons. TAO has a varying clinical presentation that can include upper eyelid retraction, restrictive strabismus, proptosis, exposure keratopathy, and optic neuropathy. In this review, we discuss the most recent literature on and the current understanding of the pathophysiology of TAO. We also review available and potential future treatment options for the management of TAO.
\end{abstract}

Keywords: Corticosteroid; Graves' orbitopathy; Rituximab; Selenium; Teprotumumab; Thyroid associated orbitopathy; Thyroid eye disease; Tocilizumab

Enhanced Digital Features To view enhanced digital features for this article go to: https://doi.org/10.6084/ m9.figshare.10335572.

N. M. Hodgson · F. Rajaii ( $\square)$

Wilmer Eye Institute, Johns Hopkins University

School of Medicine, Baltimore, MD, USA

e-mail: frajaii1@jhmi.edu

N. M. Hodgson

Department of Ophthalmology, SUNY Downstate

Medical Center, Brooklyn, NY, USA

\section{Key Summary Points}

Thyroid associated orbitopathy (TAO) is an inflammatory condition affecting the orbit and adnexa.

Patients with thyroid abnormalities should be screened for TAO and referred to a multidisciplinary TAO group early in the disease course.

Corticosteroids have been the standard of care for severe active disease since the 1960s.

Rituximab has shown mixed results in the control of active disease.

Tocilizumab has demonstrated efficacy in reducing the clinical activity score and proptosis in a small RCT.

Teprotumumab, an IGF-1R antibody in clinical trials, has shown efficacy in treating active disease and reducing proptosis in a phase III trial.

\section{INTRODUCTION}

Thyroid associated orbitopathy (TAO), also known as Graves' orbitopathy (GO) and thyroid eye disease (TED), is a disabling and disfiguring 
inflammatory condition affecting the orbit and ocular adnexa. TAO is associated with distinct clinical features, including upper eyelid retraction, restrictive strabismus, and proptosis. In certain cases, TAO can be vision threatening due to compressive optic neuropathy or corneal decompensation from exposure keratopathy.

A 2017 prospective study by the European Group on Graves' Orbitopathy (EUGOGO) determined the overall prevalence of TAO in a European population to be 10 per 10,000 persons [1]. The prevalence of moderate to severe disease was $2.96-4.45$ per 10,000 persons, while the prevalence of vision-threatening disease was lower at 0.18 per 10,000 persons. Previous crosssectional studies have found that $2 \%$ of TAO cases are vision threatening $[2,4]$. The incidence is higher in females than males in all age groups, with the 40-60 year-old age group exhibiting the highest incidence in both men and women $[3,5]$. Among patients with TAO in the Olmstead County, Minnesota population, Bartley found that 90\% had Graves' hyperthyroidism, 3\% had Hashimoto's thyroiditis, and $5 \%$ were euthyroid [5].

In addition to the morbidity associated with TAO, there is a significant impact on quality of life. Patients have difficulty with important activities of daily living, including reading and driving [6]. Further, the impact on quality of life score increases with the severity of the disease [6]. TAO has been associated with increased rates of anxiety and depression, which is partially attributable to altered cosmetic appearance [7]. Patients, particularly younger patients and women, report decreased confidence and increased social isolation, which affects mood and increases anxiety and depression $[7,8]$. It is important to recognize the psychosocial component of TAO to improve clinical care.

In this review, we discuss the natural history and course of TAO. The current understanding of the pathophysiology and mechanism of disease progression is reviewed. Additionally, we review the most recent advances in medical treatment and discuss potential future therapeutic options.

\section{METHODS}

We performed a systematic review of the PubMed database, focusing on the most recent studies and clinical trials on the medical management of TAO. This article is based on previously conducted studies and does not contain any studies with human participants or animals performed by any of the authors.

\section{NATURAL HISTORY OF TAO}

TAO manifests initially as an acute active phase. The active phase is mediated by the innate immune system. Though the pathophysiology of TAO is not yet completely understood, orbital fibroblasts are believed to be the key cells that are responsible for the soft tissue inflammation and muscle enlargement associated with the disease (Fig. 1). Activation of orbital fibroblasts that express insulin-like growth factor-1 receptor (IGF-1R) and thyrotropin receptor leads to the production of proinflammatory cytokines, including IL-6, IL-12, IL-17, IFN- $\gamma$, and TNF- $\alpha[9,10]$ (Fig. 2).

The upregulation of proinflammatory ctyokines leads to increased production of hyaluronan by orbital fibroblasts. Deposition of hyaluronan and increased adipogenesis results in enlargement of orbital soft tissues. Hypertrophy of the extraocular muscles and expansion of the orbital fat component lead to the characteristic clinical findings of strabismus, eyelid retraction, and proptosis in TAO [11-15]. The active phase of TAO occurs when inflammatory clinical characteristics are changing and

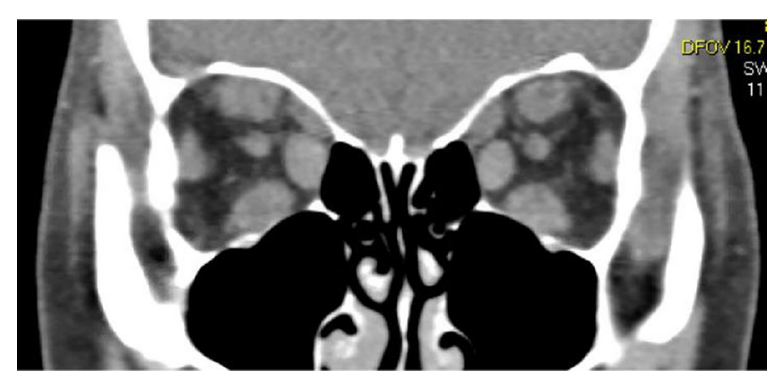

Fig. 1 Coronal CT image demonstrating enlargement of extraocular muscles in thyroid eye disease 


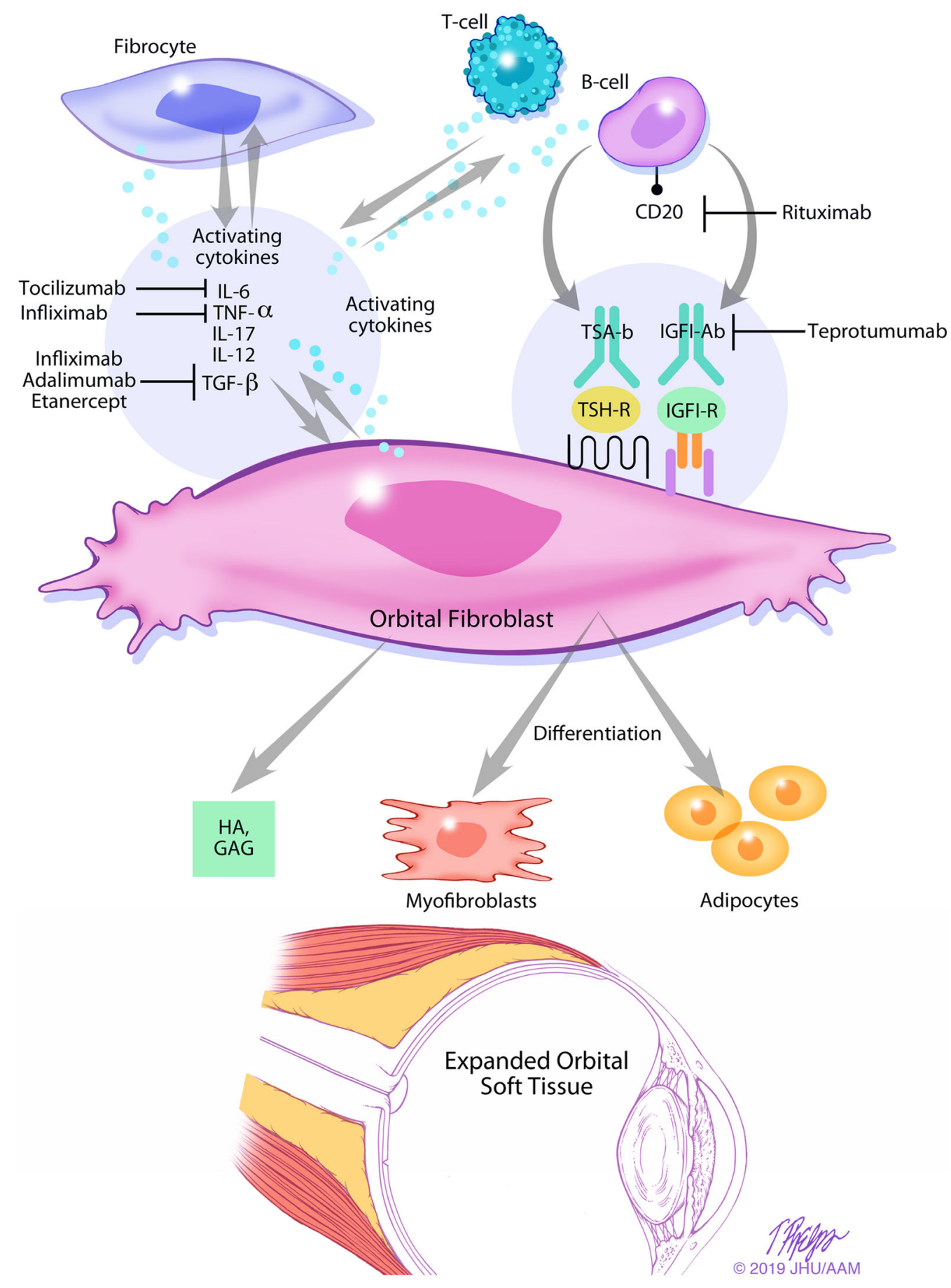

Fig. 2 Representative figure demonstrating the complex interaction of the immune system, proinflammatory cytokines, and autoantibodies on orbital fibroblasts leading to the clinical characteristics of thyroid associated orbitopathy. Sites of action of targeted therapies are displayed. Reproduced with permission from the Department of Art as Applied to Medicine, The Johns Hopkins University School of Medicine 
progressive. The active phase can last up to 24 months (as described by Rundle) and is then followed by an inactive phase [16]. The active phase can be prolonged or the disease can be reactivated by certain risk factors, including smoking [17].

The clinical features that develop in TAO vary between patients depending on the severity of their disease. The severity of TAO is determined by the presence or absence of specific clinical features, and there are many clinical severity and activity scores available to monitor disease course for clinical and research purposes, including the European Group on Graves' Orbitopathy (EUGOGO) [18, 19], VISA (Vision, Inflammation, Strabismus, Appearance) [20], NOSPECS [21, 22], and CAS (Clinical Activity Score) [23] scores. The EUGOGO classification identifies mild disease as the presence of mild lid retraction $(<2 \mathrm{~mm})$, mild exophthalmos $(<3 \mathrm{~mm})$, mild soft tissue involvement, and corneal exposure that is responsive to topical lubrication. Moderate to severe TAO is defined as lid retraction $>2 \mathrm{~mm}$, exophthalmos $>3 \mathrm{~mm}$, moderate to severe soft tissue

Table 1 Adapted from the European Group on Graves' Orbitopathy severity classification $[18,19]$

\begin{tabular}{|c|c|}
\hline \multirow{6}{*}{$\begin{array}{l}\text { Mild Graves' } \\
\text { ophthalmopathy }\end{array}$} & Lid retraction $<2 \mathrm{~mm}$ \\
\hline & Mild soft tissue involvement \\
\hline & Exophthalmos $<3 \mathrm{~mm}$ \\
\hline & $\begin{array}{l}\text { No diplopia or transient } \\
\text { diplopia }\end{array}$ \\
\hline & Exposure keratopathy \\
\hline & responsive to lubrication \\
\hline \multirow{6}{*}{$\begin{array}{l}\text { Moderate-to-severe Graves' } \\
\text { ophthalmopathy }\end{array}$} & Lid retraction $>2 \mathrm{~mm}$ \\
\hline & Moderate to severe soft \\
\hline & tissue involvement \\
\hline & Constant or inconstant \\
\hline & diplopia \\
\hline & Exophthalmos $>3 \mathrm{~mm}$ \\
\hline \multirow{3}{*}{$\begin{array}{l}\text { Sight-threatening Graves' } \\
\text { ophthalmopathy }\end{array}$} & Presence of optic neuropathy \\
\hline & Presence of corneal \\
\hline & decompensation \\
\hline
\end{tabular}

involvement, and presence of diplopia. Sightthreatening TAO is defined as presence of direct optic neuropathy or corneal breakdown (Table 1).

\section{DRIVERS OF DISEASE PROGRESSION}

Progression of TAO varies among individual patients. Males and smokers are more likely to have severe disease [24]. In a study of 59 untreated patients with mild disease observed over the course of a year, $13.5 \%$ of patients had a progressively worsening clinical course [25]. Additionally, it is known that younger patients are more likely to have orbital fat expansion while older patients are more likely to develop extraocular muscle enlargement [24, 26, 27].

Smoking is a well-known risk factor for both the progression of TAO and for more severe disease [28-30]. It has been shown that hypoxic cell culture conditions induce increased adipogenesis in orbital fibroblasts, suggesting a possible mechanism by which smoking may lead to disease progression and more severe disease states [31]. Furthermore, passive or second-hand smoke exposure has been shown to worsen Graves' disease and may worsen ophthalmopathy, as shown in childhood TAO [32, 33]. All smokers should be counseled on the association of smoking with worse clinical outcomes including risk of blindness, and the importance of smoking cessation should be emphasized at clinic visits.

Radioactive iodine (RAI) treatment has been associated with increased risk of active disease compared with thyroid medication [34, 35]. Interestingly, there was no significant difference in the rate of ophthalmopathy between RAI and thyroidectomy. Steroid prophylaxis is recommended in patients with preexisting TAO while undergoing RAI treatment.

Smoking and RAI are known risk factors for a more severe disease course; however, it is difficult to predict which patients will have more severe disease in the absence of these specific risk factors. It is important to follow patients closely after initial presentation to establish an understanding of their disease progression and 
to encourage them to notify the clinician of any significant clinical changes.

\section{TREATMENT MODALITIES}

\section{Selenium}

Selenium plays a role in the cellular redox maintenance and may reduce reactive oxygen species [36-38]. A large multicenter, randomized, double-blinded, placebo-controlled trial was performed comparing selenium 100 jig twice daily versus placebo in patients with mild TAO. The primary outcome was overall ocular improvement and quality of life measured at 6 months of treatment. There was a significant improvement in both overall ocular improvement and quality of life at 6 months which continued at 12 months with discontinuation of selenium [39]. While there was an observed benefit and no adverse events associated with selenium treatment, one controversy regarding this data is that the study was performed in areas of Europe that are known to be selenium deficient [39]. Additional studies in areas that are not selenium deficient are warranted to determine its role in the treatment of mild disease. Given its low side-effect profile, many clinicians will recommend selenium supplementation in patients with mild TAO [39].

\section{Corticosteroids}

Corticosteroids have been the mainstay firstline treatment in TED since the 1960s [40]. Corticosteroids are steroid hormones that are used to modulate the immune system and reduce inflammation in a myriad of medical conditions. In vitro studies have shown that corticosteroids reduce activation of proinflammatory cytokines in orbital fibroblasts [41, 42].

The EUGOGO classification is used to determine the need for treatment (Table 1). According to EUGOGO guidelines, intravenous glucocorticoids are advised in moderate to severe active disease, while surgical rehabilitation is recommended in moderate to severe inactive disease [18, 19]. Sight-threatening TED
Table 2 Adapted from the Clinical Activity Score (CAS). A score of 3 or greater is indicative of active disease that would be responsive to immunosuppressive therapy [23]

Spontaneous orbital pain 1

Pain on up or down gaze 1

Eyelid erythema 1

Conjunctival erythema 1

Eyelid edema 1

Chemosis 1

Caruncular inflammation $\quad 1$

Increase in proptosis of $2 \mathrm{~mm}$ or more between 1 and 1 3 months

Decrease in visual acuity of 1 or more lines between $1 \quad 1$ and 3 months

Decrease in motility of 5 or more degrees between 1 and 3 months

demands immediate intervention, while mild disease can be monitored without intervention. The clinical activity score can be used to determine if the disease is active or inactive (Table 2) [23]. Cases of moderate to severe active disease should proceed with intravenous glucocorticoid therapy followed by rehabilitative surgery once the disease becomes inactive. In cases of sightthreatening disease, an initial trial of two weeks of intravenous glucocorticoid therapy is recommended, and urgent decompression should be performed if there is no improvement.

The recommended dosing for orbital inflammation without diplopia is $500 \mathrm{mg}$ weekly for 6 weeks followed by $250 \mathrm{mg}$ weekly for 6 weeks. If diplopia is present, the recommended dosing schedule is $750 \mathrm{mg}$ weekly for 6 weeks followed by $500 \mathrm{mg}$ weekly for 6 weeks total [43]. The total cumulative dose of corticosteroids should not exceed $8 \mathrm{~g}$. Comparative studies of intravenous versus oral corticosteroids have demonstrated a significant benefit from intravenous administration. Intravenous administration was found to be more effective and required fewer surgeries than oral therapy $[43,44]$. When the clinical course has stabilized, rehabilitative surgery should be considered to 
address clinical symptoms including proptosis, diplopia, and lid retraction.

Contraindications to glucocorticoid therapy include hepatitis or liver disease, uncontrolled diabetes, hypertension, and severe cardiovascular disease. Patients are often comanaged with a primary care physician or endocrinologist to monitor glucose levels and blood pressure while on treatment.

\section{Rituximab}

Rituximab is a humanized chimeric monoclonal anti-CD20 antibody. Extraocular muscles and orbital fat have been shown to have lymphocytic infiltration during the active phase of TED. Additionally, TSH receptor antibodies (TRAb) have been shown to be elevated in the onset of active disease [45]. Rituximab targets B cells, which leads to $\mathrm{B}$ cell depletion and therefore may affect autoantibodies to the TSH receptor.

Stan et al. conducted a prospective randomized controlled study with 21 patients randomized to two $1000 \mathrm{mg}$ rituximab infusions or two saline infusions two weeks apart. The primary outcome was a reduction in CAS score at 24 weeks. This study did not meet its targeted sample size, and demonstrated no significant benefit of rituximab compared to placebo [46]. Another double-blinded, randomized trial by Salvi et al. demonstrated improved outcomes with rituximab compared to IV methylprednisolone. In this study, 32 patients were randomized to receive rituximab (1000 mg twice or $500 \mathrm{mg}$ once) or IV methylprednisolone (7.5 g). At 24 weeks, all rituximab patients had improved, compared with $69 \%$ of the methyprednisolone patients [47].

Rituximab is typically well tolerated. The most common side effects are hypotension, transient fevers, and pruritus. Serious adverse events including dysthyroid optic neuropathy, vasculitis, and sudden death have been reported $[46,48]$. Results of these trials are contradictory, and no other randomized controlled studies have been performed to further elucidate the utility of anti-CD20 therapy in thyroid eye disease.

\section{Tocilizumab}

Tocilizumab is a humanized monoclonal antiIL- 6 antibody. It has been shown that fibroblasts increase the secretion of IL-6, leading to increased adipogenesis. Small case series have also demonstrated a reduction in inflammation on biopsy of orbital fat [49] and a reduction in TSI [50] following tocilizumab therapy.

Perez-Moreiras performed an interventional, nonrandomized study of 18 patients with active thyroid eye disease refractory to intravenous corticosteroids. Patients were administered tocilizumab therapy and were found to have reductions in CAS score, TSI levels, proptosis $(72 \%)$, and extraocular motility (83\%). These results are limited by the small and nonrandomized design of this study [51].

The Tocilizumab for Graves' Orbitopathy study group conducted a masked randomized control trial of 32 patients randomized to intravenous tocilizumab $8 \mathrm{mg} / \mathrm{kg}$ at $0,4,8$, and 12 weeks versus placebo. All patients had moderate to severe disease that did not respond to corticosteroids. There was a significant difference in the number of patients who achieved the primary outcome, a change in CAS score of at least 2, with $93.3 \%$ of patients in the tocilizumab group achieving this endpoint compared to only $58.8 \%$ of controls. In addition, there was a $1.5 \mathrm{~mm}$ reduction in proptosis in tocilizumab patients compared to no change in proptosis in controls. Thus, there are promising data supporting the use of tocilizumab to treat TAO [52].

\section{Teprotumumab}

Teprotumumab is a human monoclonal IGF-1R antibody. Though IGF-1R is a widely expressed receptor, IGF-1R expression is increased on fibroblasts from the orbit, skin, and thyroid of patients with Graves' disease. The upregulation of IGF-1R and the TSHR in orbital fibroblasts has been proposed to lead to the accumulation of hyaluronan within orbital fat and muscle $[10,53,54]$.

Teprotumumab has been evaluated in clinical trials. Smith et al. conducted a phase 2 
placebo-controlled, double-blinded study enrolling patients with moderate to severe active TED [55]. 88 patients were enrolled in this study and were administered infusions of teprotumumab or placebo every 3 weeks for 24 weeks. The patients were stratified by smoking status, though there were more smokers in the placebo group than the active drug group. This study demonstrated a reduction in both clinical activity score and proptosis at 24 weeks. Infusions were associated with few severe adverse events, including diarrhea and Hashimoto's encephalopathy, which were thought to be associated with the study drug. Additional side effects included nausea and muscle spasms, and diabetic patients were noted to have developed hyperglycemia.

This study was particularly significant, as this is the first medical therapy that has demonstrated a reduction in proptosis. Douglas determined that patients with higher baseline proptosis had a greater response to teprotumumab than patients with lower baseline proptosis (mean reduction 3.7 vs. $2.6 \mathrm{~mm}$ ) [53]. Further, there was improvement in proptosis in all gender- and smoking-stratified groups. The results of the phase II trial are somewhat limited by the higher number of smokers in the placebo group.

More recently, data from the phase III trial have been presented. The phase III trial confirmed the results of the phase II trial. There was a significant difference in proptosis reduction between the teprotumumab group and the placebo group at weeks $6,12,18$, and 24 . Data from secondary endpoints are yet to be presented at the time of this review $[56,57]$.

Teprotumumab is a promising medical therapy for the treatment of TAO. Further studies will be needed to evaluate the results of this therapy in patients with inactive disease and to compare its efficacy to those of tocilizumab, rituximab, and other immunomodulators.

\section{Orbital Radiotherapy}

Orbital radiotherapy is a well-established treatment for TAO. Orbital lymphocytes and fibroblasts are sensitive to radiation treatment.
Standard dosing is a total of 20 Gy bilaterally over two weeks, though some studies indicate a lower dose may be effective in appropriate patients [58]. Older studies have shown that orbital radiotherapy is as effective as corticosteroid treatment and that a combination of steroid and radiation is more effective than either modality alone $[59,60]$.

A recent study was conducted with 40 patients with moderate to severe disease resistant to high-dose corticosteroid therapy. Patients were given $20 \mathrm{~Gy}$ in 10 fractions with concurrent $500 \mathrm{mg}$ methylprednisolone weekly. $32.5 \%$ of patients had a regression in diplopia and there was a median reduction in CAS score of 2 . Treatment was well tolerated, with $7.5 \%$ of patients developing cataract and $12.5 \%$ of patients developing dry eye [61]. Additionally, Bartalena et al. conducted a randomized trial of 48 patients which confirmed that combined therapy is superior to steroids alone in patients with moderate to severe disease [62].

Additionally, low-dose radiotherapy has been shown to be effective. Cardoso et al. conducted a study where 18 patients received $10 \mathrm{~Gy}$ over 10 weeks. All patients in the study showed improvements in ocular pain, motility, and edema [63]. Additionally, Kahaly performed a randomized blinded trial comparing radiation doses. The study showed that low dose (1 Gy/day) and high dose (2 Gy/day) were equally effective. Low-dose radiation therapy may reduce side effects, including retinopathy, cataract formation, and secondary tumors [58].

Orbital radiotherapy is an important adjuvant to steroid therapy in the appropriate patient with active moderate or severe TAO. The side effects are limited, and multiple studies have consistently demonstrated the treatment's efficacy.

\section{Surgical Management}

Despite appropriate medical therapy, nearly $20 \%$ of patients with TAO will require some form of surgical therapy. The EUGOGO consensus guidelines propose urgent orbital decompression in vision-threatening disease if there is a poor response to corticosteroids. There 
are multiple treatment algorithms for surgical decompression, including balanced decompression, medial and floor decompression, lateral wall decompression, and orbital fat decompression. Surgical management often varies based on surgeon preference and training, though results from balanced decompressions appear to be greater. A recent retrospective multivariate analysis determined that greater preoperative proptosis, balanced decompression, and history of orbital radiation were predictors of a greater reduction in proptosis after decompression [64]. In cases of compressive optic neuropathy, posteromedial decompression is recommended $[65,66]$. Comparative studies of orbital decompression techniques would help define an optimal surgical management algorithm.

Rehabilitative surgery is postponed until the disease is inactive and clinical measurements are stable. The goal of rehabilitative surgery is to restore the patient to as close to the predisease state as possible. Rehabilitative surgical options include orbital decompression for severe proptosis or aesthetic concerns, strabismus surgery, followed by upper and lower lid retraction repair. Again, surgical techniques can differ based on surgeon preference and have varying degrees of success. A recent survey of members of the American Society of Ophthalmic Plastic and Reconstructive Surgeons demonstrated that the majority of survey respondents prefer a balanced medial and lateral decompression as their first approach [67]. More research is needed to compare the outcomes of different surgical approaches and determine an optimal decompression protocol.

\section{FUTURE MEDICAL THERAPIES}

Additional therapies have been proposed and are in varying stages of study for efficacy in TAO. Methotrexate has been used to effectively treat autoimmune diseases such as rheumatoid arthritis. Its mechanism of action is the inhibition of the enzyme dihydrofolate reductase, which interferes with DNA and protein synthesis and in lower doses has anti-inflammatory properties. A retrospective review demonstrated an improvement in both CAS score and motility restriction with methotrexate therapy [68]. Prospective data have not been presented.

TNF- $\alpha$ is known to be upregulated in TAO. Several studies have evaluated the use of TNF- $\alpha$ antagonists. In a retrospective review of adalimumab in TAO, six of 10 patients showed a reduction in inflammation; however, three patients had an increase in inflammation [69]. Infliximab has been shown to be beneficial in single case reports [70]. It is difficult to interpret the utility of anti-TNF therapy with limited small-scale studies.

Cyclic peptides may be another promising avenue for future therapy. Holthoff et al. established a mouse model that was immunized with a recombinant adenovirus expressing the human TSH receptor (TSHR) [71]. Cyclic peptides were created that replicated the tertiary structure of the TSHR leucine-rich repeat domain. The cyclic peptides were able to stabilize TSH receptorbinding antibodies, reducing thyroid hyperplasia and improving retro-orbital fibrosis [71]. This is an encouraging result in an animal model, and future confirmatory animal studies may lead to translation to clinical therapy for TAO.

Other potential targets on the inflammatory cascade include the interleukin pathway. IL-17 is known to be upregulated in many autoimmune diseases [72], and targeted therapies against IL-17 have shown promise in psoriasis and ankylosing spondylitis [73, 74]. Further, IL15 and IL-17 are upregulated in patients' tears in active disease compared with inactive disease. A targeted therapy to IL-15 or IL-17 may have a role in the management of TAO [75]. Other avenues of potential study should include targeting hyaluronan synthesis, adipogenesis, and fibroblast differentiation.

\section{CONCLUSIONS}

Thyroid associated orbitopathy is a debilitating and vision-threatening disease. The pathophysiology is yet to be completely elucidated; however, TAO is understood to be an inflammatory disease. Activation of proinflammatory cytokines results in increased hyaluronan 
production by orbital fibroblasts, leading to the classic clinical manifestations of TAO.

Patients with thyroid abnormalities should be screened and educated about the signs and symptoms of TAO. Upon diagnosing TAO, a referral to a TAO specialist multidisciplinary group should be made early in the course of the disease per the Thyroid Eye Disease Amsterdam Declaration Implementation Group UK (TEAMeD) guidelines [76]. A multidisciplinary group including oculoplastic surgery, strabismus surgery, and neuro-ophthalmology can closely monitor patients for disease progression and implement appropriate medical and surgical therapy.

Oral selenium supplementation is recommended in mild disease, given the low risk and potential benefit. Corticosteroids remain the mainstay first-line treatment in active moderate-to-severe and vision-threatening disease. Intravenous corticosteroids have been shown to be superior to oral administration. Orbital radiotherapy is an important adjuvant to corticosteroid treatment, and low-dose radiation may be adequate in the treatment of TAO.

Biologic agents have demonstrated potential as therapeutic alternatives or adjuncts in the treatment of TAO. The data on rituximab is contradictory and thus will require future analyses to determine its efficacy. Tocilizumab studies did demonstrate an improvement in disease state; however, the trials were limited by size.

The results of the teprotumumab clinical trials are of great interest. Teprotumumab is the first therapy to demonstrate an improvement in proptosis and therefore may reduce the need for orbital decompression. We anticipate that teprotumumab will play an important role in the medical management of TAO.

Surgical management is still required in cases of severe vision-threatening disease that is refractory to medical management, and as restorative treatment when the disease is quiescent. The management of TAO has evolved significantly and will continue to do so. We anticipate significant improvements in the management of TAO patients with the use of upcoming biologic agents and targeted therapies.

\section{ACKNOWLEDGMENTS}

Funding. No funding or sponsorship was received for this study or the publication of this article.

Authorship. All named authors meet the International Committee of Medical Journal Editors (ICMJE) criteria for authorship for this article, take responsibility for the integrity of the work as a whole, and have given their approval for this version to be published.

Disclosures. Fatemeh Rajaii is supported by the National Eye Institute of Health under award number K08EY027093 and is a consultant for Horizon Pharmaceuticals. Nickisa M. Hodgson has nothing to disclose.

Compliance with Ethics Guidelines. This article is based on previously conducted studies and does not contain any studies with human participants or animals performed by any of the authors.

Open Access. This article is licensed under a Creative Commons Attribution-NonCommercial 4.0 International License, which permits any non-commercial use, sharing, adaptation, distribution and reproduction in any medium or format, as long as you give appropriate credit to the original author(s) and the source, provide a link to the Creative Commons licence, and indicate if changes were made. The images or other third party material in this article are included in the article's Creative Commons licence, unless indicated otherwise in a credit line to the material. If material is not included in the article's Creative Commons licence and your intended use is not permitted by statutory regulation or exceeds the permitted use, you will need to obtain permission directly from the copyright holder. To view a copy of this licence, visit http://creativecommons.org/licenses/by$\mathrm{nc} / 4.0 /$. 


\section{REFERENCES}

1. Perros P, Hegedus L, Bartalena L, et al. Graves' orbitopathy as a rare disease in Europe: a European Group on Graves' Orbitopathy (EUGOGO) position statement. Orphanet J Rare Dis. 2017;12:72.

2. Tanda ML, Piantanida E, Liparulo L, Veronesi G, Lai A, Sassi L, et al. Prevalence and natural history of Graves' orbitopathy in a large series of patients with newly diagnosed Graves' hyperthyroidism seen at a single center. J Clin Endocrinol Metab. 2013;98: 1443-9. https://doi.org/10.1210/jc.2012-3873.

3. Perros P, Žarković M, Azzolini C, Ayvaz G, Baldeschi L, Bartalena L, et al. PREGO (presentation of Graves' orbitopathy) study: changes in referral patterns to European Group On Graves' Orbitopathy (EUGOGO) centres over the period from 2000 to 2012. Br J Ophthalmol. 2015;99:1531-5.

4. Laurberg P, Berman DC, Pederson IB, et al. Incidence and clinical presentation of moderate to severe Graves' orbitopathy in a Danish population before and after iodine fortification of salt. J Clin Endocrinol Metab. 2012;97(7):2325-32.

5. Bartley GB. The epidemiologic characteristics and clinical course of ophthalmopathy associated with autoimmune thyroid disease in Olmsted County, Minnesota. Trans Am Ophthalmol Soc. 1994;92: 477-588.

6. Park JJ, Sullivan TJ, Mortimer RH, et al. Assessing quality of life in Australian patients with Graves' ophthalmopathy. Br J Ophthalmol. 2004;88(1): 75-8.

7. Wickwar S, McBain HB, Ezra DG, et al. What are the psychosocial outcomes of treatment for thyroid eye disease? A systematic review. Thyroid. 2014;24(9): 1407-18.

8. Coulter I, Frewin S, Krassas GE, Perros P. Psychological implications of Graves' orbitopathy. Eur J Endocrinol. 2007;157(2):127-31.

9. Shan SJ, Douglas RS. The pathophysiology of thyroid eye disease. J Neuroophthalmol. 2014;34(2): 177-85.

10. Naik VM, Naik MN, Goldberg RA, et al. Immunopathogenesis of thyroid eye disease: emerging paradigms. Surv Ophthalmol. 2010;55(3): 215-26.

11. Dik WA, Virakul S, van Steensel L. Current perspectives on the role of orbital fibroblasts in the pathogenesis of Graves' ophthalmopathy. Exp Eye Res. 2016;142:83-91.
12. Krieger CC, Neumann S, Place RF, et al. Bidirectional TSH and IGF-1 receptor cross talk mediates stimulation of hyaluronan secretion by Graves' disease immunoglobins. J Clin Endocrinol Metab. 2015;100(3):1071-7.

13. Smith TJ, Koumas L, Gagnon A, Bell A, Sempowski GD, Phipps RP, Sorisky A. Orbital fibroblast heterogeneity may determine the clinical presentation of thyroid-associated ophthalmopathy. J Clin Endocrinol Metab. 2002;87(1):385-92.

14. Kaback LA, Smith TJ. Expression of hyaluronan synthase messenger ribonucleic acids and their induction by interleukin-1beta in human orbital fibroblasts: potential insight into the molecular pathogenesis of thyroid-associated ophthalmopathy. J Clin Endocrinol Metab. 1999;84(11):4079-84.

15. Verity DH, Rose GE. Acute thyroid eye disease (TED): principles of medical and surgical management. Eye (Lond). 2013;27(3):308-19.

16. Rundle FF, Wilson CW. Development and course of exophthalmos and ophthalmoplegia in Graves' disease with special reference to the effect of thyroidectomy. Clin Sci. 1945;5(3-4):177-94.

17. Cawood TJ, Moriarty P, O'Farrelly C, et al. Smoking and thyroid-associated ophthalmopathy: a novel explanation of the biological link. J Clin Endocrinol Metab. 2007;92(1):59-64 (Epub 2006 Oct 17).

18. Bartalena L, Baldeschi L, Dickinson AJ, et al. Consensus statement of the European Group on Graves' Orbitopathy (EUGOGO) on management of Graves' orbitopathy. Thyroid. 2008;18:333-46.

19. Bartalena L, Baldeschi L, Boboridis K, European Group on Graves' Orbitopathy (EUGOGO), et al. The 2016 European Thyroid Association/European Group on Graves' Orbitopathy Guidelines for the Management of Graves' Orbitopathy. Eur Thyroid J. 2016;5(1):9-26.

20. Dolman PJ, Rootman J. VISA classification for Graves orbitopathy. Ophthalmic Plast Reconstr Surg. 2006;22(5):319-24.

21. Werner SC. Classification of the eye changes of Graves' disease. J Clin Endocrinol Metab. 1969;29: 782-4.

22. Werner SC. Modification of the classification of the eye changes of Graves' disease. Am J Ophthalmol. 1977;83:725-7.

23. Mourits MP, Koornneef L, Wiersinga WM, et al. Clinical criteria for the assessment of disease activity in Graves' ophthalmopathy: a novel approach. Br J Ophthalmol. 1989;73(8):639-44. 
24. Kendler DL, Lippa J, Rootman J. The initial clinical characteristics of Graves' orbitopathy vary with age and sex. Arch Ophthalmol. 1993;111(2):197-201.

25. Perros P, Crombie AL, Kendall-Taylor P. Natural history of thyroid associated ophthalmopathy. Clin Endocrinol (Oxf). 1995;42(1):45-50.

26. Ben Simon GJ, Katz G, Zloto O, et al. Age differences in clinical manifestation and prognosis of thyroid eye disease. Graefes Arch Clin Exp Ophthalmol. 2015;253(12):2301-8.

27. Nunery WR. Ophthalmic Graves' disease: a dual theory of pathogenesis. Ophthalmol Clin N Am. 1991;4:73-87.

28. Prummel MF, Wiersinga WM. Smoking and risk of Graves' disease. JAMA. 1993;269(4):479-82.

29. Stan MN, Bahn RS. Risk factors for development or deterioration of Graves' ophthalmopathy. Thyroid. 2010;20(7):777-83.

30. Thornton J, Kelly SP, Harrison RA, Edwards R. Cigarette smoking and thyroid eye disease: a systematic review. Eye (Lond). 2007;21(9):1135-45.

31. Chng CL, Lai OF, Chew CSM, et al. Hypoxia increases adipogenesis and affects adipocytokine production in orbital fibroblasts-a possible explanation of the link between smoking and Graves' ophthalmopathy. Int J Ophthalmol. 2014;7(3): 403-7.

32. Krassas GE, Segni M, Wiersinga WM. Childhood Graves' ophthalmopathy: results of a European questionnaire study. Eur J Endocrinol Eur Fed Endocr Soc. 2005;153:515-21.

33. Krassas GE, Perros P. Prevention of thyroid associated ophthalmopathy in children and adults: current views and management of preventable risk factors. Pediatr Endocrinol Rev. 2007;4(3):218-24.

34. Acharya SH, Avenell A, Philip S, et al. Radioiodine therapy (RAI) for Graves' disease (GD) and the effect on ophthalmopathy: a systematic review. Clin Endocrinol (Oxf). 2008;69(6):943-50. https://doi. $\operatorname{org} / 10.1111 /$ j.1365-2265.2008.03279.

35. Bartalena L, Marcocci C, Bogazzi F, et al. Relation between therapy for hyperthyroidism and the course of Graves' ophthalmopathy. N Engl J Med. 1998;338:73-8.

36. Marino M, Menconi F, Rotondo Dottore G, et al. Selenium in Graves hyperthyroidism and orbitopathy. Ophthalmic Plast Reconstr Surg. 2018;34(4S Suppl 1):S105-10.
37. Ventura M, Melo M, Carrilho F. Selenium and thyroid disease: from pathophysiology to treatment. Int J Endocrinol. 2017;2017:1297658.

38. Rotondo Dottore G, Leo M, Casini G, et al. Antioxidant actions of selenium in orbital fibroblasts: a basis for the effects of selenium in Graves' orbitopathy. Thyroid. 2017;27(2):271-8.

39. Marcocci C, Kahaly GJ, Krassas GE. Selenium and the course of mild Graves' orbitopathy. N Engl J Med. 2011;364(20):1920-31.

40. Hales IB, Thoma ID, et al. Treatment of thyroid ophthalmopathy with corticoid analogues. Australas Ann Med. 1962;11:113-7.

41. Burnstine MA, Elner SG, Strieter RM, et al. Orbital fibroblast interleukin-6 gene expression and immunomodulation. Ophthalmic Plast Reconstr Surg. 1999;15(5):306-11.

42. Burnstine MA, Elner SG, Elner V. Orbital fibroblast chemokine modulation: effects of dexamethasone and cyclosporin A. Br J Ophthalmol. 1998;82(3): 318-22.

43. Kahaly GJ, Pitz S, Hommel G, et al. Randomized, single blind trial of intravenous versus oral steroid monotherapy in Graves' orbitopathy. J Clin Endocrinol Metab. 2005;90(9):5234-40.

44. Aktaran S, Akarsu E, Erbağci I, et al. Comparison of intravenous methylprednisolone therapy vs. oral methylprednisolone therapy in patients with Graves' ophthalmopathy. Int J Clin Pract. 2007;61(1):45-51.

45. Salvi M, Vannucchi G, Campi I, et al. Efficacy of rituximab treatment for thyroid-associated ophthalmopathy as a result of intraorbital B-cell depletion in one patient unresponsive to steroid immunosuppression. Eur J Endocrinol. 2006;154(4):511-7.

46. Stan MN, Garrity JA, Carranza Leon BG, et al. Randomized controlled trial of rituximab in patients with Graves' orbitopathy. J Clin Endocrinol Metab. 2015;100(2):432-41.

47. Salvi M, Vannucchi G, Curro N, et al. Efficacy of B-cell targeted therapy with rituximab in patients with active moderate to severe Graves' orbitopathy: a randomized controlled study. J Clin Endocrinol Metab. 2015;100:422-31.

48. Khanna D, Chong KK, Afifiyan NF, et al. Rituximab treatment of patients with severe, corticosteroidresistant thyroid-associated ophthalmopathy. Ophthalmology. 2010;117(133-139):e2. 
49. Sy A, Eliasieh K, Silkiss RZ. Clinical response to tocilizumab in severe thyroid eye disease. Ophthal Plast Reconstr Surg. 2017;33(3):e55-7.

50. Russel DJ, Wagner LH, Seiff SR. Tocilizumab as a steroid sparing agent for the treatment of Graves' orbitopathy. Am J Ophthalmol Case Rep. 2017;7: 146-8.

51. Pérez-Moreiras JV, Alvarez-López A, Gómez EC. Treatment of active corticosteroid-resistant Graves' orbitopathy. Ophthal Plast Reconstr Surg. 2014;30(2):162-7. https://doi.org/10.1097/IOP. 0000000000000037.

52. Perez-Moreiras JV, Gomez-Reino JJ, Maneiro JR, et al. Tocilizumab in Graves Orbitopathy study group. Efficacy of tocilizumab in patients with moderate-to-severe corticosteroid-resistant Graves orbitopathy: a randomized clinical trial. AJO. 2018;195:181-90.

53. Douglas RS. Teprotumumab, an insulin-like growth factor-1 receptor antagonist antibody, in the treatment of active thyroid eye disease: a focus on proptosis. Eye (Lond). 2019;33(2):183-90.

54. Smith TJ. New advances in understanding thyroidassociated ophthalmopathy and the potential role for insulin-like growth factor-I receptor. F1000Res. 2018;7:134.

55. Smith TJ, Kahaly GJ, Ezra DG, et al. Teprotumumab for thyroid-associated ophthalmopathy. N Engl J Med. 2017;376(18):1748-61.

56. Douglas RS. New data from phase 3 teprotumumab trial (OPTIC) shows dramatic reduction in proptosis, or eye bulging, the main cause of morbidity in active thyroid eye disease (TED). Biospace. April 26, 2019.

57. Patel A, Yang H, Douglas RS. A new era in the treatment of thyroid eye disease. AJO. 2019;208: 281-8.

58. Kahaly GJ, Rösler HP, Pitz S, Hommel G. Low-versus high-dose radiotherapy for Graves' ophthalmopathy: a randomized, single blind trial. J Clin Endocrinol Metab. 2000;85(1):102-8.

59. Prummel MF, Mourits MP, Blank L, Berghout A, Koornneef L, Wiersinga WM. Randomized doubleblind trial of prednisone versus radiotherapy in Graves' ophthalmopathy. Lancet. 1993;342(8877): 949-54.

60. Marcocci C, Bartalena L, Bogazzi F, et al. Orbital radiotherapy combined with high dose systemic glucocorticoids for Graves' ophthalmopathy is more effective than radiotherapy alone: results of a prospective randomized study. J Endocrinol Investig. 1991;14(10):853-60.

61. Nicosia L, Reverberi C, Agolli L, et al. Orbital radiotherapy plus concomitant steroids in moderate-to-severe Graves' ophthalmopathy: good results after long-term follow-up. Int J Endocrinol Metab. 2019;17(1):e84427.

62. Bartalena L. What to do for moderate-to-severe and active Graves' orbitopathy if glucocorticoids fail? Clin Endocrinol (Oxf). 2010;73(2):149-52.

63. Cardoso CC, Giordani AJ, Wolosker AM, et al. Protracted hypofractionated radiotherapy for Graves' ophthalmopathy: a pilot study of clinical and radiologic response. Int J Radiat Oncol Biol Phys. 2012;82(3):1285-91.

64. Wu CY, Niziol LM, Musch DC, Kahana A. Thyroidrelated orbital decompression surgery: a multivariate analysis of risk factors and outcomes. Ophthal Plast Reconstr Surg. 2017;33(3):189-95.

65. McCann JD, Goldberg RA, Anderson RL, Burroughs JR, Ben Simon GJ. Medial wall decompression for optic neuropathy but lateral wall decompression with fat removal for non vision-threatening indications. Am J Ophthalmol. 2006;141(5):916-7.

66. Metson R, Pletcher SD. Endoscopic orbital and optic nerve decompression. Otolaryngol Clin $\mathrm{N}$ Am. 2006;39:551-61.

67. DeParis SW, Tian J, Rajaii F. Practice patterns in orbital decompression surgery among American Society of Ophthalmic Plastic and Reconstructive Surgery members. Ophthalmol Ther. 2019;8:541-8.

68. Strianese D, Iuliano A, Ferrara M, et al. Methotrexate for the treatment of thyroid eye disease. J Ophthalmol. 2014;2014:128903.

69. Ayabe R, Rootman DB, Hwang CJ, et al. Adalimumab as steroid-sparing treatment of inflammatory-stage thyroid eye disease. Ophthalmic Plast Reconstr Surg. 2014;30(5):415-9.

70. Durrani OM, Reuser TQ, Murray PI. Infliximab: a novel treatment for sight-threatening thyroid associated ophthalmopathy. Orbit. 2005;24(2): 117-9.

71. Holthoff HP, Li Z, Fabbender J, et al. Cyclic peptides for effective treatment in a long-term model of Graves disease and orbitopathy in female mice. Endocrinology. 2017;158(7):2376-90.

72. Rajaii F, McCoy AN, Smith TJ. Cytokines are both villains and potential therapeutic targets in thyroidassociated ophthalmopathy. From bench to bedside. Expert Rev Ophthalmol. 2014;9(3):227-34. 
73. Chiricozzi A, Krueger JG. IL-17 targeted therapies for psoriasis. Expert Opin Investig Drugs. 2013;22(8):993-1005.

74. Baeten D, Baraliakos X, Braun J, et al. Anti-interleukin-17A monoclonal antibody secukinumab in treatment of ankylosing spondylitis: a randomised, double-blind, placebo-controlled trial. Lancet. 2013;382(9906):1705-13.

75. Chen Q. The expression of interleukin-15 and interleukin-17 in tears and orbital tissues of Graves ophthalmopathy patients. J Cell Biochem. 2019;120(4):6299-303.

76. Mellington FE, Dayan CM, Dickinson AJ, Thyroid Eye Disease Amsterdam Declaration Implementation Group (TEAMeD), et al. Management of thyroid eye disease in the United Kingdom: a multicentre thyroid eye disease audit. Eye Disease Amsterdam Implementation Group UK. Orbit. 2017;36(3):159-69. 